\title{
Pretreatment serum levels of IL-1 receptor antagonist and IL-4 are predictors of overall survival in multiple myeloma patients treated with bortezomib
}

\author{
Damian Mikulski ${ }^{1,2}$, Paweł Robak ${ }^{2,3}$, Ewelina Perdas ${ }^{1}$, Edyta Węgłowskaa ${ }^{4}$, Aleksandra Łosiewiczi ${ }^{1}$ Izabela Dróżḋ் ${ }^{5}$, \\ Dariusz Jarych $^{6}$, Małgorzata Misiewicz ${ }^{2,7}$, Janusz Szemraj', Wojciech Fendler', Tadeusz Robak $2,7^{*}$
}

1. Department of Biostatistics and Translational Medicine, Medical University of Lodz, 93-215 Lodz, Poland; damian.mikulski@stud.umed.lodz.pl (D.M.); ewelina.perdas@umed.lodz.pl (E.P.), aleksandra.losiewicz@stud.umed.lodz.pl (A.Ł); wojciech.fendler@umed.lodz.pl (W.F.)

2. Copernicus Memorial Hospital, Lodz 93-510, Poland; pawel.robak@umed.lodz.pl (P.R.); malgorzata.misiewicz@umed.lodz.pl (M.M.); robaktad@csk.umed.lodz.pl (T.R.)

3. Department of Experimental Hematology, Medical University of Lodz, Lodz 93-510, Poland

4. Laboratory of Personalized Medicine, Bionanopark, Lodz 93-465; , e.weglowska@bionanopark.pl (E.W.);

5. Department of Clinical Genetics, Medical University of Lodz, 92-213 Lodz, Poland; izabela.drozdz@umed.lodz.pl (I.D.)

6. Laboratory of Virology, Institute of Medical Biology, Polish Academy of Sciences, 93-232 Lodz, Poland; djarych@cbm.pan.pl (D.J.)

7. Department of Hematology, Medical University of Lodz, 93-510 Lodz

8. Department of Medical Biochemistry, Medical University of Lodz, 92-215 Lodz, Poland

* Correspondence: robaktad@csk.umed.lodz.pl

Orcid Tadeusz Robak 0000-0002-3411-6357

Orcid Damian Mikulski 0000-0002-2806-2583

Orcid Paweł Robak 000-0002-6078-5415

Orcid Dariusz Jarych: 0000-0003-1303-7163

\begin{abstract}
Multiple myeloma (MM) is characterized by the malignant proliferation of monoclonal plasma cells in the bone marrow with an elevation in monoclonal paraprotein, renal impairment, hypercalcemia, lytic bony lesions, and anemia. Immune cells and associated cytokines play a significant role in MM growth, progression, and dissemination. While some cytokines and their clinical significance are well described in MM biology, others remain relatively unknown. The aim of the present study was to assess the impact of pretreatment serum levels of 27 selected cytokines on progression-free survival (PFS) and overall survival (OS) in MM patients before first-line therapy with bortezomib-based regimens. Serum cytokine levels were assayed with a Bio-Rad Bio-Plex Pro Human Cytokine 27-Plex Assay on the MAGPIX Multiplex Reader and the Bio-Plex® 200 System (Bio-Rad) including IL-1 $\beta$, IL-1Ra, IL-2, IL-4, IL-5, IL-6, IL-7, IL-8, IL-9, IL-10, IL-12, IL-13, IL-15, IL-17, Eotaxin, FGF, G-CSF, GM-CSF, IFN- $\gamma$, IP-10, MCP-1, MIP-1 $\alpha$, MIP-1 $\beta$, PDGF-BB, RANTES, TNF- $\alpha$, and VEGF. A total of $61 \mathrm{MM}$ patients were examined. Most patients received a bortezomib, cyclophosphamide and dexamethasone (VCD) chemotherapy regimen. In the final multivariate model, IL-13 cytokine level (HR 0.1411, 95\% CI: 0.0240-0.8291, p = 0.0302), and ASCT (HR 0.3722, 95\% CI: 0.1826-0.7585, p=0.0065) significantly impacted PFS. Furthermore, ASCT (HR 0.142, 95\% CI: 0.046-0.438, $\mathrm{p}=0.0007$ ), presence of bone disease at diagnosis (HR 3.826, 95\% CI: 1.471-9.949, $\mathrm{p}=0.0059)$ and two cytokine levels- IL-1Ra (HR 1.017, 95\% CI: 1.004-1.030, p= 0.0091) and IL-4 (HR 0.161, 95\% CI: 0.037-0.698, $\mathrm{p}=0.0147$ ) were independent predictors of OS. Three clusters of MM patients were identified with different cytokine profiles. In conclusion, serum pretreatment levels of IL-13 and IL-4 are predictors of better PFS and OS, respectively, whereas IL-1Ra pretreatment levels negatively impact OS in MM patients treated with bortezomib-based chemotherapy. Cytokine signature profile may have a potential influence on the outcome of patients treated with bortezomib.
\end{abstract}


Keywords: bortezomib, IL-13, IL-1Ra, IL-4, multiple myeloma, OS, PFS

\section{Introduction}

Multiple myeloma (MM) is a neoplasm characterized by aberrant expansion of monoclonal plasma cells with bone marrow involvement, plasma renal impairment, hypercalcemia, lytic bony lesions, and anemia [1,2]. It is the second-most prevalent blood cancer in the United States and Europe. According to the WHO, 176404 new MM cases and 117077 deaths due to MM occurred worldwide in 2020 [3]. Multiple myeloma usually happens around the age of 60 and is more common in men than women $[4,5]$.

Multiple myeloma is an incurable disease characterized by substantial morbidity and mortality [5]. However, survival of patients with MM has improved significantly over the past 15 years following the introduction of several novel therapeutic agents, including proteasome inhibitors (PI), immunomodulating agents (IMiD) and monoclonal antibodies [5,6]. In addition, high-dose chemotherapy with autologous stem-cell transplantation (ASCT) significantly prolongs overall survival (OS) in younger patients. The MM treatment options have never been so broad, and new biomarkers are urgently needed to identify an adequate treatment regimen for a particular patient.

A crucial role in the treatment of MM is played by proteasome inhibitors, of which bortezomib was the first-in-class selective and reversible example. It was approved for medical use in the United States in 2003 and in the European Union in 2004 [7]. Bortezomib demonstrates antiproliferative and antitumor activity, and its use has been a breakthrough in treating MM [8]. However, most patients treated with bortezomib-based regimens eventually relapse and often demonstrate multiple drug resistance [9].

Immune cells and associated cytokines play a crucial role in the growth, progression, and dissemination of MM. While the roles of some cytokines, such as IL- 6 , TGF- $\beta$, and IL-1 $\beta$, are well described in the MM biology, those of others remain unclear [10-13]. Previous reports indicated that bortezomib treatment is associated with significantly reduced serum levels of many cytokines and chemokines, including IL- $1 \alpha / \beta$, IL-3, IL-4, IL-5, IL-6, IL-8, IL-12p40, IL-12p70, IFN- $\gamma$, TNF- $\alpha$, MIP-1 $\alpha / \beta$, GM-CSF, CXCL1, RANTES, VEGF, Eotaxin, and IL-13 [11-14]. Our previous study investigated circulating cytokine, growth factor, and chemokine levels and their association with selected clinical and laboratory disease characteristics [11]. Several cytokines were found to be involved in the pathogenesis of $\mathrm{MM}$ and some were useful in predicting treatment response in bortezomib-treated patients; in particular, higher levels of MIP-1 $\alpha$ and lower levels of MIP-1 $\beta$ and IL-9 were associated with better response to treatment, while higher IL-1Ra and IL-8 levels were associated with osteolytic bone symptoms.

The aim of the present study was to assess the impact of pretreatment serum levels of 27 selected cytokines on progression-free survival (PFS) and overall survival (OS) in MM patients treated with bortezomib-based regimens as first-line therapy.

\section{Materials and Methods}

\subsection{Patients}

The study group comprised 61 patients with MM treated at the Department of Hematology, Copernicus Memorial Hospital, Lodz, Poland, from February 2016 to September 2019. All of the patients received bortezomib-based treatment as first-line therapy. Response to treatment and progression events were classified according to the International Myeloma Working Group [14,15]. The study was conducted according to good clinical and laboratory practice rules and the principles of the Declaration of Helsinki. All procedures were approved by the local ethical committee (The Ethical Committee of the Medical University of Lodz, No. RNN/103/16/KE).

\subsection{Cytokine Analysis}


The method of cytokine analysis has previously been described in detail [12]. Brifly, patients with preexisting conditions (allergy/infections) that may cause changes in the cytokine profile were excluded before blood sample collection. After the collection of whole blood, it was left undisturbed at room temperature for 30 minutes. The samples were then centrifuged at $2000 \times \mathrm{g}$ for 10 minutes. Serum samples from all studied patients were stored at $-80^{\circ} \mathrm{C}$.

Serum cytokine levels were assayed with Bio-Rad Bio-Plex Pro Human Cytokine 27-Plex Assay on the MAGPIX Multiplex Reader and the Bio-Plex® 200 System (Bio-Rad) as described previously [12]. Briefly, the Luminex ${ }^{\circ} \times \mathrm{xMP} ®$ technology based on immunoassay methods is capable of simultaneously quantifying 27 targets: IL-1 $\beta$, IL-1 receptor antagonist (IL-1Ra), IL-2, IL-4, IL-5, IL-6, IL-7, IL-8, IL-9, IL-10, IL-12, IL-13, IL-15, IL-17, Eotaxin (CCL11), fibroblast growth factor (FGF), granulocyte colony-stimulating factor (G-CSF), granulocyte-macrophage colony-stimulating factor (GM-CSF), interferon gamma (IFN- $\gamma)$, IFN- $\gamma$-induced protein 10 (IP-10), monocyte chemoattractant protein 1 (MCP-1), macrophage inflammatory protein- (MIP) $1 \alpha$ and MIP-1 $\beta$, platelet-derived growth factor BB (PDGF-BB), RANTES (Regulated on Activation, Normal T Expressed and Secreted, CCL5), tumor necrosis factor $\alpha$ (TNF- $\alpha)$, and vascular endothelial growth factor (VEGF).

The concentration of each cytokine was extrapolated from the calibration curve (individual for each cytokine) determined independently for each experiment (each plate). All samples were analyzed in duplicate.

\subsection{Statistical analysis}

Nominal variables were expressed as percentages and analyzed using the Chi-square test with appropriate corrections if needed: the Yates correction for continuity or Fisher's exact test. The normality of the distribution of continuous variables was verified with the Shapiro-Wilk test. Continuous variables were presented as mean \pm standard deviation (SD). ANOVA with post hoc Tukey's test was used to compare cytokine concentrations between identified MM patient clusters. Heatmap and hierarchical agglomerative clustering were used to evaluate clusters of patients with similar plasma cytokine profiles.

Survival analysis was conducted using a Kaplan-Meier estimate with univariate and multivariate Cox's proportional hazards models, as well as the log-rank test. Optimal cutoff values were determined using Cutoff Finder [16]. In Cox's models, cytokine concentrations were divided by 10 to interpret coefficient values more straightforward. Analyses were conducted using Statistica 13.1 (TIBCO, Palo Alto, CA, USA). P values < 0.05 were considered statistically significant.

\section{Results}

\subsection{Patients' Characteristics}

The demographic, clinical, and laboratory characteristics of the MM patients enrolled in the study are presented in Table 1 . The group comprised 32 men and 29 women with a mean age of $61.9 \pm 11.3$ years (range: $38.3-83.7$ ). The distribution according to the ISS stage I-III was $27.9 \%, 21.3 \%$ and $47.5 \%$, respectively (missing data- $3.3 \%$ ). The predominant isotype of monoclonal protein was IgG kappa (52.5\%). Regarding CRAB symptoms, most patients (59\%) presented with bone disease, 34.4\% displayed $\mathrm{Hb}<10$ $\mathrm{g} / \mathrm{dL}$, hypercalcemia was noted in $19.7 \%$, and renal failure was observed in $16.4 \%$ of patients. Cytogenetics data were available for 33 patients $(54.1 \%)$; the most common abnormalities were $\operatorname{amp}(1 \mathrm{q})$ followed by $\operatorname{del}(13 \mathrm{q})$, with frequencies of $18(54.5 \%)$ and 8 $(24.5 \%)$, respectively. The majority of patients $(80.3 \%)$ received a bortezomib, cyclophosphamide, and dexamethasone (VCD) chemotherapy regimen. The objective response rate with primary induction treatment for all patients was $83.6 \%$, including $60.6 \%$ $\geq$ VGPR. Complete response was achieved by 24 (39.3\%) of patients. More than one third $(37.7 \%)$ of patients underwent ASCT. 
Table 1. The characteristics of patients treated with bortezomib-based therapy. Data are presented as frequency and percentage (\%) unless otherwise specified.

\begin{tabular}{|c|c|}
\hline Characteristics & Total \\
\hline Number of patients & $61(100)$ \\
\hline \multirow{2}{*}{ Gender } & M: $32(52.5)$ \\
\hline & F: $29(47.5)$ \\
\hline Age at diagnosis & $61.9 \pm 11.3$ \\
\hline mean + SD (range) & $(38.3-83.7)$ \\
\hline \multicolumn{2}{|l|}{ Bortezomib regimen: } \\
\hline VCD & $49(80.3)$ \\
\hline VMP & $5(8.2)$ \\
\hline VTD & $4(6.6)$ \\
\hline Other & $3(4.9)$ \\
\hline \multicolumn{2}{|l|}{ Paraprotein } \\
\hline $\operatorname{IgG}$ & $33(54)$ \\
\hline $\operatorname{Ig} A$ & $14(23)$ \\
\hline LCD & $14(23)$ \\
\hline Bone disease at diagnosis & $36(59)$ \\
\hline Calcium $>2.75 \mathrm{mmol} / \mathrm{l}$ at diagnosis & $12(19.7)$ \\
\hline $\mathrm{HB}<10 \mathrm{~g} / \mathrm{dL}$ at diagnosis & $21(34.4)$ \\
\hline Creatinine $>2 \mathrm{mg} / \mathrm{dL}$ at diagnosis & $10(16.4)$ \\
\hline \multirow{3}{*}{ International Staging System (ISS) } & I -17 (27.9) \\
\hline & $\mathrm{II}-13(21.3)$ \\
\hline & III-29 (47.5) \\
\hline Beta2-microglobuline increased $(>3 \mathrm{mg} / \mathrm{L})$ & $40(65.6)$ \\
\hline $\mathrm{LDH}>240 \mathrm{U} / \mathrm{L}$ & $7(11.5)$ \\
\hline Cytogenetics* & $\mathrm{N}=33$ \\
\hline $\mathrm{t}(11 ; 14)$ & $1(3)$ \\
\hline $\mathrm{t}(4 ; 14)$ & $5(15.2)$ \\
\hline $\mathrm{t}(14 ; 16)$ & 0 \\
\hline $\mathrm{t}(14 ; 20)$ & 0 \\
\hline $\operatorname{del}(17 p)$ & $3(9.1)$ \\
\hline $\operatorname{amp}(1 q)$ & $18(54.5)$ \\
\hline $\operatorname{del}(13 q)$ & $8(24.2)$ \\
\hline
\end{tabular}

${ }^{*}$ Cytogenetics data were available for 33 patients (54.1\%). Abbreviations: LCD - light chain disease; VCD - bortezomib, cyclophosphamide, and dexamethasone; VD-bortezomib and dexamethasone; VMP - bortezomib, melphalan, and prednisone; VTD-bortezomib, thalidomide, and dexamethasone.

\subsection{Prognostic impact of clinical variables}

Data on PFS and on OS was available for 61 patients. The median PFS and overall survival OS of the cohort were 13.1 and 51.8 months, respectively. Univariate Cox regression analyses of clinical variables for PFS and OS are summarized in Table 2. Only autologous stem cell transplantation (ASCT) during the treatment schedule was clinical 
factor influencing both PFS (HR 0.33, 95\% CI: $0.17-0.64, \mathrm{p}=0.001$ ) and OS (HR 0.23, 95\% CI: $0.09-0.62, \mathrm{p}=0.004)$. The corresponding Kaplan-Meier plots are shown in Figure 1.

Table 2. Univariate Cox regression analyses of basic clinical variables for progression-free survival and overall survival.

\begin{tabular}{|c|c|c|c|c|c|c|c|c|c|c|}
\hline \multirow{3}{*}{ Variable } & \multicolumn{5}{|c|}{ PFS } & \multicolumn{5}{|c|}{ OS } \\
\hline & \multirow{2}{*}{ Coefficient } & \multirow{2}{*}{$p$} & \multirow{2}{*}{ HR } & \multicolumn{2}{|c|}{$95 \% \mathrm{CI}$} & \multirow{2}{*}{ Coefficient } & \multirow{2}{*}{$p$} & \multirow{2}{*}{ HR } & \multicolumn{2}{|c|}{$95 \% \mathrm{CI}$} \\
\hline & & & & Lower & Upper & & & & Lower & Upper \\
\hline ISS III & -0.13 & 0.680 & 0.88 & 0.48 & 1.60 & 0.78 & 0.060 & 2.18 & 0.97 & 4.90 \\
\hline ASCT & -1.11 & 0.001 & 0.33 & 0.17 & 0.64 & -1.46 & 0.004 & 0.23 & 0.09 & 0.62 \\
\hline $\begin{array}{l}\mathrm{HB}<10 \mathrm{~g} / \mathrm{dL} \\
\text { at diagnosis }\end{array}$ & 0.12 & 0.720 & 1.13 & 0.59 & 2.17 & 0.12 & 0.778 & 1.13 & 0.49 & 2.58 \\
\hline Calcium $>2.75 \mathrm{mmol} / \mathrm{l}$ at diagnosis & 0.46 & 0.213 & 1.58 & 0.77 & 3.23 & -0.40 & 0.464 & 0.67 & 0.23 & 1.95 \\
\hline $\begin{array}{c}\text { Creatinine }>2 \mathrm{mg} / \mathrm{dL} \\
\text { at diagnosis }\end{array}$ & -0.48 & 0.323 & 0.62 & 0.24 & 1.60 & 0.07 & 0.893 & 1.08 & 0.36 & 3.18 \\
\hline Bone disease & 0.64 & 0.054 & 1.89 & 0.99 & 3.61 & 0.61 & 0.156 & 1.85 & 0.79 & 4.30 \\
\hline Age $>70$ & 0.34 & 0.323 & 1.41 & 0.71 & 2.80 & 0.63 & 0.118 & 1.87 & 0.85 & 4.10 \\
\hline
\end{tabular}
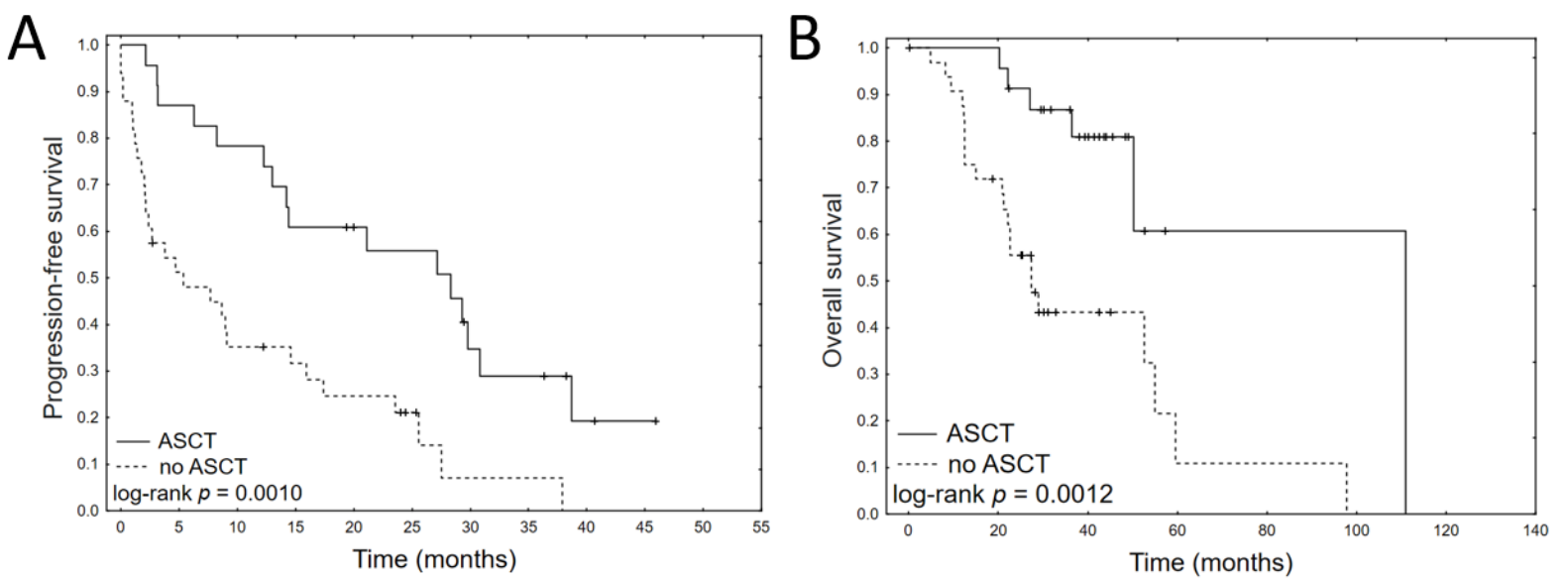

Figure 1. Kaplan-Meier plots of progression-free survival (A) and overall survival (B) for ASCT in MM patients treated with bortezomib.

\subsection{Prognostic impact of cytokine levels}

Univariate Cox regression analyses of pretreatment serum cytokine levels for PFS and OS are provided in Table 3. Only IL-13 serum level significantly impacted PFS (HR 0.1398, 95\% CI: 0.0272- 0.7189, p = 0.0185). Regarding OS, five cytokines were significant in univariate analyses: IL-13 (HR 0.0121, 95\% CI: 0.0004- 0.3271, p = 0.0087), IL-1ra (HR 1.0527, CI:1.0177-1.0889, p = 0.0029), IL-4 (HR 0.1899, CI: 0.0401-0.9000, p = 0.0364), IL-7 (HR 0.6313, CI:0.3988-0.9993, p=0.0497) and PDGF-BB (HR 0.9963, CI: 0.9930-0.9997 p= 0.0316). The Kaplan-Meier plots for dichotomized cytokine levels are provided in Figure 2.

The cytokines found to be significant in univariate analysis were entered into a multivariate model building with a stepwise backward Akaike information criterion (AIC) elimination procedure, together with well-established prognostic factors (ISS III, ASCT, age $>70$, and presence of bone disease). Briefly, an optimized model is identified by creating multiple models, initially including all selected variables. The variable with the highest $p$-value is eliminated at each step, and an AIC value is calculated for the new model; this process is repeated until no variables remain. The final multivariate models 
for PFS and OS with the lowest overall AICs are given in Table 4. The analysis confirmed that cytokine levels retained their importance in the simultaneous context of the clinical prognostic factors. The final model for PFS consisted of two variables: IL-13 cytokine level (HR 0.141, 95\% CI: 0.024-0.829, $p=0.0302$ ), and ASCT (HR 0.3722, 95\% CI: 0.1826-0.7585, $\mathrm{p}=0.0065)$. The final model for OS included ASCT (HR 0.142, 95\% CI: 0.046-0.438, $\mathrm{p}=0.0007$ ), presence of bone disease at diagnosis (HR 3.826, 95\% CI: 1.471-9.949, $\mathrm{p}=0.0059)$ and two cytokine levels- IL-1Ra (HR 1.017, 95\% CI: 1.0004-1.030, p $=0.0091)$ and IL-4 (HR 0.161, 95\% CI: 0.037-0.698, p = 0.0147).

Table 3. Univariate Cox regression analyses of cytokine levels for progression-free survival and overall survival. Cytokine concentrations $(\mathrm{pg} / \mathrm{ml})$ were divided by 10 to allow a more straightforward interpretation of coefficient values.

\begin{tabular}{|c|c|c|c|c|c|c|c|c|c|c|}
\hline \multirow{3}{*}{ Cytokine } & \multicolumn{5}{|c|}{ PFS } & \multicolumn{5}{|c|}{ OS } \\
\hline & \multirow{2}{*}{ Coefficient } & \multirow{2}{*}{$p$} & \multirow{2}{*}{ HR } & \multicolumn{2}{|c|}{$95 \%$ CI } & \multirow{2}{*}{ Coefficient } & \multirow{2}{*}{$p$} & \multirow{2}{*}{ HR } & \multicolumn{2}{|c|}{$95 \%$ CI } \\
\hline & & & & Lower & Upper & & & & Lower & Upper \\
\hline Eotaxin & 0.0022 & 0.9558 & 1.0022 & 0.9279 & 1.0824 & -0.0954 & 0.0620 & 0.9090 & 0.8224 & 1.0048 \\
\hline FGF basic & -0.0825 & 0.6462 & 0.9208 & 0.6475 & 1.3096 & -0.2964 & 0.2059 & 0.7435 & 0.4697 & 1.1769 \\
\hline G-CSF & -0.0009 & 0.8658 & 0.9991 & 0.9882 & 1.0101 & 0.0002 & 0.9755 & 1.0002 & 0.9852 & 1.0155 \\
\hline GM-CSF & -1.7105 & 0.0694 & 0.1808 & 0.0285 & 1.1456 & $-4,9713$ & 0.0643 & 0.0069 & 0.00004 & 1.3441 \\
\hline IFN- $\gamma$ & -0.0230 & 0.8837 & 0.9773 & 0.7184 & 1.3296 & -0.1371 & 0.5175 & 0.8719 & 0.5756 & 1.3206 \\
\hline IL-10 & -0.0005 & 0.9984 & 0.9995 & 0.6270 & 1.5934 & 0.0049 & 0.0531 & 1.0049 & 0.9999 & 1.0099 \\
\hline IL-12 (p70) & 0.6213 & 0.3417 & 1.8613 & 0.5172 & 6.6991 & 0.3990 & 0.7549 & 1.4903 & 0.1217 & 18.2445 \\
\hline IL-13 & -1.9675 & 0.0185 & 0.1398 & 0.0272 & 0.7189 & -4.4187 & 0.0087 & 0.0121 & 0.0004 & 0.3271 \\
\hline IL-15 & 0.0138 & 0.5837 & 1.0139 & 0.9652 & 1.0650 & -0.0110 & 0.7869 & 0.9890 & 0.9131 & 1.0713 \\
\hline IL-17 & -0.0975 & 0.5954 & 0.9071 & 0.6329 & 1.3001 & -0.4021 & 0.1243 & 0.6689 & 0.4006 & 1.1170 \\
\hline IL-1ra & 0.0012 & 0.8111 & 1.0012 & 0.9917 & 1.0107 & 0.0514 & 0.0029 & 1.0527 & 1.0177 & 1.0889 \\
\hline IL- $1 \beta$ & -1.6824 & 0.1799 & 0.1859 & 0.0159 & 2.1737 & -1.3066 & 0.5299 & 0.2707 & 0.0046 & 15.9668 \\
\hline IL-2 & -0.3222 & 0.6034 & 0.7246 & 0.2149 & 2.4429 & -1.2680 & 0.1562 & 0.2814 & 0.0488 & 1.6234 \\
\hline IL-4 & -0.1722 & 0.7859 & 0.8418 & 0.2430 & 2.9165 & -1.6615 & 0.0364 & 0.1899 & 0.0401 & 0.9000 \\
\hline IL-5 & 0.1369 & 0.2194 & 1.1467 & 0.9217 & 1.4268 & -0.0654 & 0.6848 & 0.9367 & 0.6829 & 1.2847 \\
\hline IL-6 & 0.0328 & 0.9321 & 1.0334 & 0.4860 & 2.1970 & -0.2548 & 0.6893 & 0.7751 & 0.2223 & 2.7027 \\
\hline IL-7 & 0.0450 & 0.7981 & 1.0460 & 0.7411 & 1.4762 & -0.4600 & 0.0497 & 0.6313 & 0.3988 & 0.9993 \\
\hline IL-8 & 0.1698 & 0.3739 & 1.1850 & 0.8151 & 1.7229 & 0.0972 & 0.7127 & 1.1021 & 0.6570 & 1.8487 \\
\hline IL-9 & 0.0217 & 0.1296 & 1.0219 & 0.9937 & 1.0509 & -0.0149 & 0.3157 & 0.9852 & 0.9570 & 1.0143 \\
\hline IP-10 & -0.0005 & 0.9984 & 0.9995 & 0.6270 & 1.5934 & 0.0049 & 0.0531 & 1.0049 & 0.9999 & 1.0099 \\
\hline MCP-1 & 0.0856 & 0.4049 & 1.0894 & 0.8906 & 1.3326 & -0.2965 & 0.0992 & 0.7435 & 0.5226 & 1.0575 \\
\hline $\mathrm{MIP}-1 \alpha$ & -0.1895 & 0.3722 & 0.8273 & 0.5457 & 1.2545 & 0.0639 & 0.7731 & 1.0660 & 0.6903 & 1.6461 \\
\hline MIP-1 $\beta$ & 0.1054 & 0.0990 & 1.1112 & 0.9804 & 1.2595 & -0.0463 & 0.4618 & 0.9548 & 0.8441 & 1.0800 \\
\hline PDGF-BB & -0.0012 & 0.2900 & 0.9988 & 0.9965 & 1.0011 & -0.0037 & 0.0316 & 0.9963 & 0.9930 & 0.9997 \\
\hline RANTES & -0.0007 & 0.1152 & 0.9993 & 0.9984 & 1.0002 & -0.0010 & 0.0572 & 0.9990 & 0.9981 & 1.0000 \\
\hline TNF- $\alpha$ & -0.1612 & 0.3417 & 0.8511 & 0.6105 & 1.1866 & -0.3491 & 0.1528 & 0.7053 & 0.4371 & 1.1382 \\
\hline VEGF & 0.0022 & 0.7425 & 1.0022 & 0.9890 & 1.0157 & -0.0395 & 0.1177 & 0.9613 & 0.9149 & 1.0100 \\
\hline
\end{tabular}


A

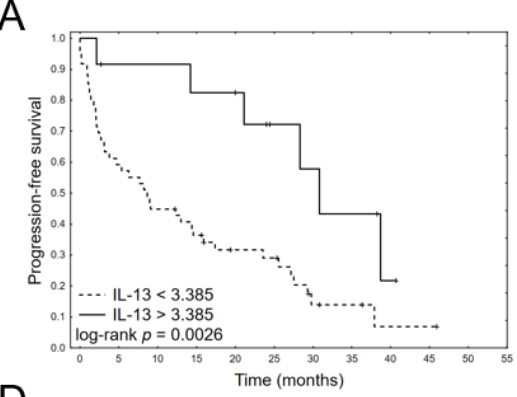

D

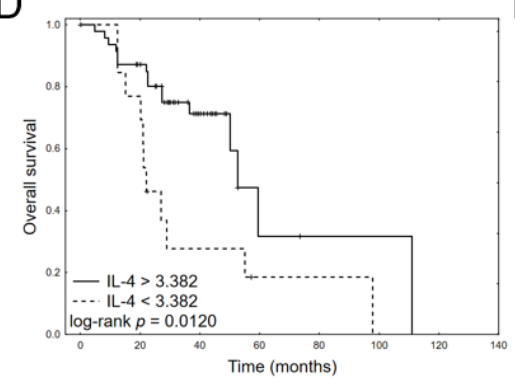

B

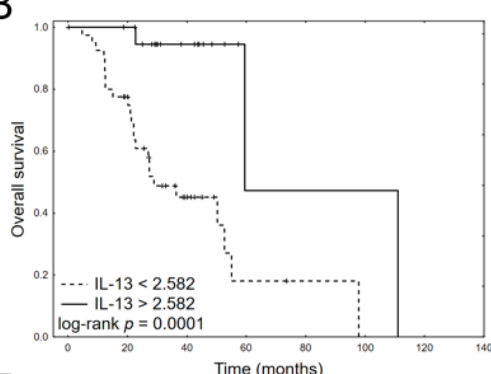

$\mathrm{E}$

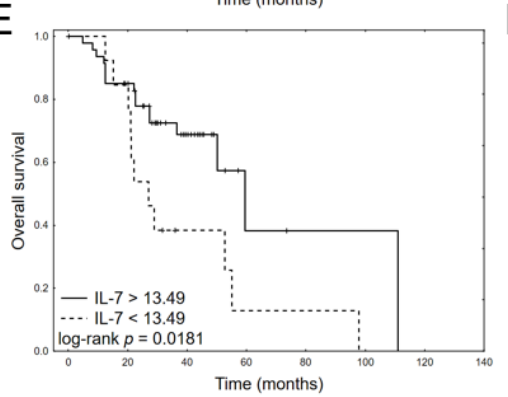

C

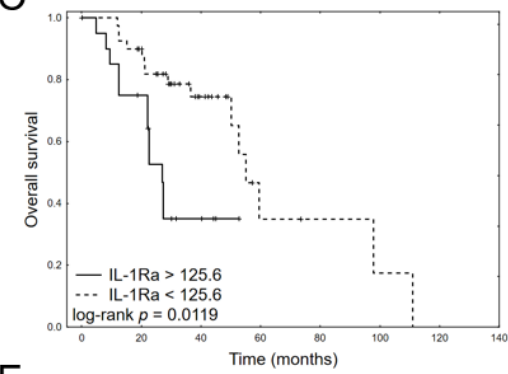

F

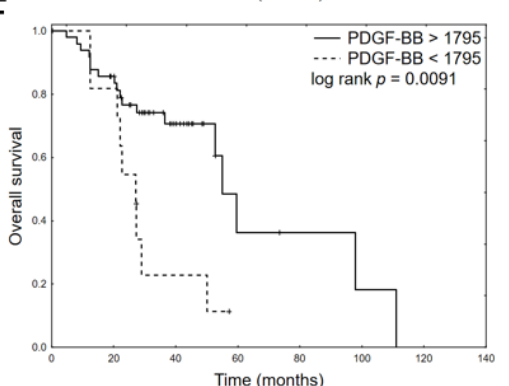

Figure 2. Kaplan-Meier plots for dichotomized significant cytokines in univariate analysis- PFS- IL-13 (A) and OS: IL-13 (B), IL-1Ra (C), IL-4 (D), IL-7 (E), PDGF-BB (F).

The cytokines found to be significant in univariate analysis were entered into a multivariate model building with a stepwise backward Akaike information criterion (AIC) elimination procedure, together with well-established prognostic factors (ISS III, ASCT, age $>70$, and presence of bone disease). Briefly, an optimized model is identified by creating multiple models, initially including all selected variables. The variable with the highest p-value is eliminated at each step, and an AIC value is calculated for the new model; this process is repeated until no variables remain. The final multivariate models for PFS and OS with the lowest overall AICs are given in Table 4. The analysis confirmed that cytokine levels retained their importance in the simultaneous context of the clinical prognostic factors. The final model for PFS consisted of two variables: IL-13 cytokine level (HR 0.141, 95\% CI: 0.024-0.829, p = 0.0302), and ASCT (HR 0.3722, 95\% CI: $0.1826-0.7585, \mathrm{p}=0.0065)$. The final model for OS included ASCT (HR 0.142, 95\% CI: $0.046-0.438, \mathrm{p}=0.0007$ ), presence of bone disease at diagnosis (HR 3.826, 95\% CI: 1.471-9.949, $\mathrm{p}=0.0059)$ and two cytokine levels- IL-1Ra (HR 1.017, 95\% CI: 1.0004-1.030, p $=0.0091)$ and IL-4 (HR 0.161, 95\% CI: 0.037-0.698, p = 0.0147).

Table 4. Final multivariate Cox regression analyses for PFS and OS of MM patients. Cytokine concentrations (pq/ml) were divided by 10 to allow a more straightforward interpretation of coefficient values.

\begin{tabular}{|c|c|c|c|c|c|}
\hline \multirow{7}{*}{ Variable } & \multirow{2}{*}{ Coefficient } & $p$ & \multirow{2}{*}{ HR } & \multicolumn{2}{|c|}{$95 \%$ CI } \\
\cline { 4 - 6 } & & & & Lower & Upper \\
\hline \multicolumn{7}{|c|}{ OS } \\
\hline IL-13 & -1.958 & 0.0302 & 0.1411 & 0.0240 & 0.8291 \\
\hline ASCT & -0.494 & 0.0065 & 0.3722 & 0.1826 & 0.7585 \\
\hline \multicolumn{7}{|c|}{} \\
\hline IL-1Ra & 0.017 & 0.0091 & 1.017 & 1.004 & 1.030 \\
\hline IL-4 & -1.828 & 0.0147 & 0.161 & 0.037 & 0.698 \\
\hline ASCT & -0.975 & 0.0007 & 0.142 & 0.046 & 0.438 \\
\hline bone disease & 0.671 & 0.0059 & 3.826 & 1.471 & 9.949 \\
\hline
\end{tabular}


A two-way hierarchical cluster analysis was performed to classify cytokine patterns among multiple myeloma patients (Figure 3A). Six cytokines were excluded from the analysis due to high missing values $(>15 \%)$ : GM-CSF $(n=41$ values below limit of detection), IL-12(p70) ( $n=39)$, IL-10 ( $n=27)$, IL-15 $(n=25)$, IFN- $\gamma(n=18)$ and VEGF $(n=10)$. One patient (ID 50) was excluded, as eleven cytokines in his sample were below the limit of detection.

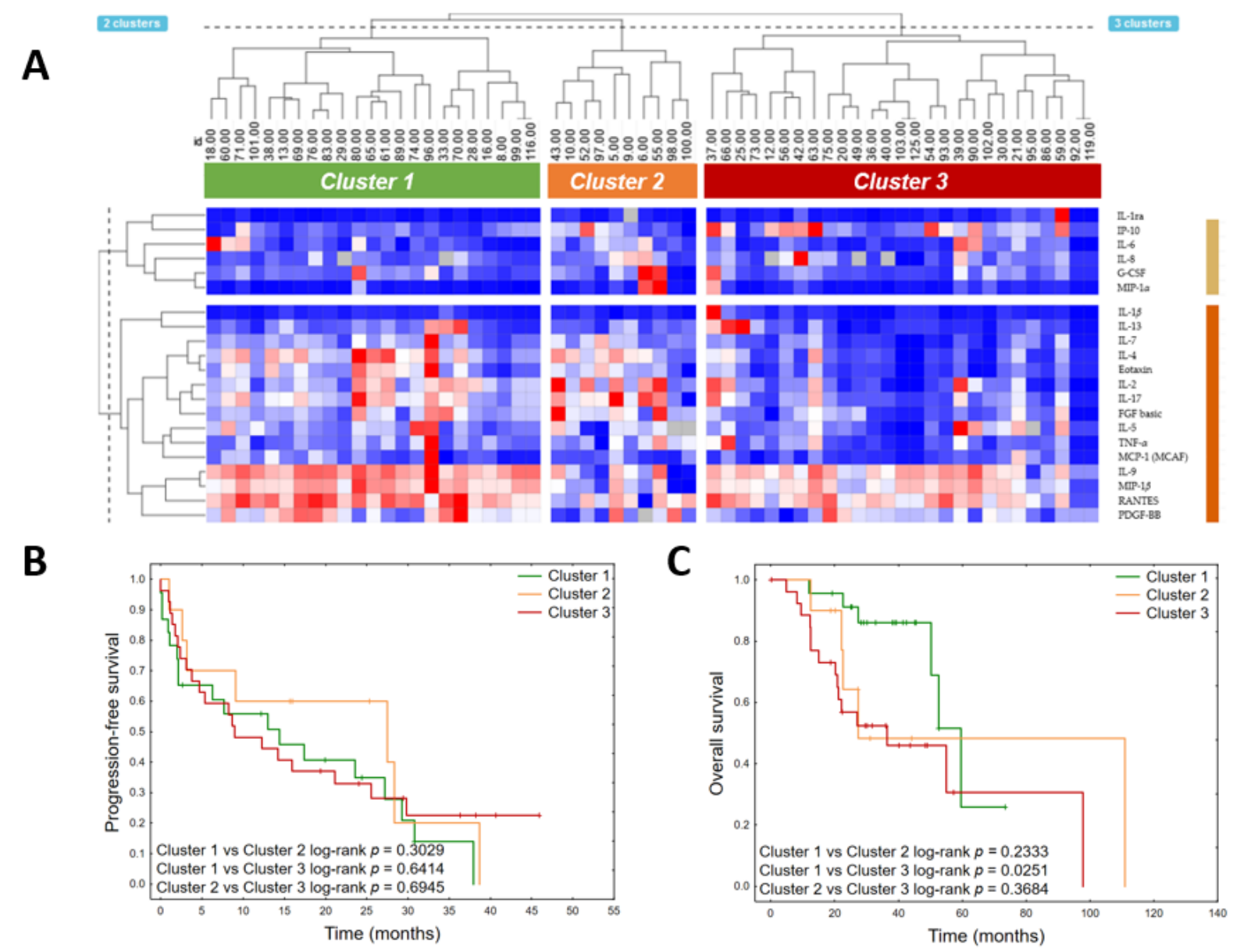

Figure 3. Heatmap with hierarchical clustering performed on cytokine values (A) to identify multiple myeloma patient groups. Expression levels of individual cytokines are represented by shades of blue to red in the central heatmap, with the highest values in dark red and the lowest in dark blue. Kaplan-Meier plots of progression-free survival (B) and overall survival (C) each identified cluster of patients.

Two main clusters of cytokines were identified and three clusters of patients. The first cytokine clusters consisted of IL-1ra, IP-10, IL-6, IL-8, G-CSF and MIP-1 $\alpha$. The second consisted of IL-1 $\beta$, IL-13, IL-7, IL-4, Eotaxin, IL-2, IL-17, FGF basic, IL-5, TNF- $\alpha$, MCP-1 (MCAF), IL-9, MIP-1 $\beta$, RANTES and PDGF-BB. The detailed level of each cytokine in particular clusters is provided in Table $S 1$.

The patients in the first cluster were more frequently ISS III stage ( $p=0.0287$ ). Comparison of clinical variables between clusters is detailed in Table S2. Furthermore, patients in the third cluster had significantly shorter OS (HR 2.988 95\% CI: 1.1454-7.7444, $\mathrm{p}=0.0252$ ) (Figure 3B, C).

\section{Discussion}

Previous studies have showed that different cytokines take part in the pathogenesis, progression, and prognosis of $\mathrm{MM}[12,17]$. The present study evaluated the prognostic significance of 27 cytokine serum levels in 61 previously-untreated MM patients receiving bortezomib-based regimens as first-line treatment. Furthermore, three clusters of patients were identified with different overall survival based on cluster analysis. 
We observed that a higher serum pretreatment level of IL-13 is an independent predictor of longer PFS in MM patients treated with bortezomib-based chemotherapy. IL-13 is an anti-inflammatory Th2-type cytokine that suppresses the cytotoxic actions of macrophages, inhibits the production of pro-inflammatory cytokines, and plays a significant role in various inflammatory diseases [18]. Moreover, IL-13 plays a role in human osteoclast formation in a lymphocyte-dependent manner [19]. On the other hand, Di Lulo et al. [20] strongly suggest a role for IL-13 in MM progression through upregulation of adhesion molecules and IL- 6 secretion by bone marrow mesenchymal stromal cells, which promotes MM cell growth. Previous studies have found that IL-13 upregulates VCAM-1 expression on endothelial cells and increases adhesion molecule expression and IL-6 secretion in fibroblasts [21-23]. These observations suggest that bone marrow-derived mesenchymal stem cells (BM-MSCs) can be targets for IL-13 in MM [20]. IL-13 increases adhesive molecule expression and IL-6 secretion by BM-MSCs; these cells demonstrate a similar response to IL-13 stimulation as other cells, including stromal fibroblasts [22]. It has been documented previously that IL-13 is involved in the stimulation of macrophages for antitumor activity. The IL13-activated macrophage subset is characterized by abundant amounts of macrophage C-type lectin receptors (CLRs) $[24,25]$. In the preclinical study IL13 inhibited T-cell lymphoma and ovarian adenocarcinoma development in mice through the conversion of tumor-supporting macro-phages to cytotoxic effectors [25]. The prognostic value of serum IL-13 level has been evaluated previously in other hematological malignancies. Özyörük et al [26] report higher serum IL-13 levels in children with lymphoma diagnosed with Hodgkin lymphoma or Burkitt's lymphoma; however, unlike the present study, they did not find this cytokine to have any prognostic significance.

Among the 27 tested cytokines, IL-1Ra, IL-4, IL-7, IL-13 and PDGF-BB were found to be predictors of OS in MM patients treated with bortezomib-based regimens in univariate models, and IL-1Ra and IL-4 maintained this significance in the multivariate model. However, among the MM patients treated with bortezomib, only IL-4 predicted longer OS in both the univariate and multivariate models: Patients with higher level of IL-4 had longer OS (Fig. 2). In normal conditions, IL-4 induces TH2 cell, B-cell, mast cell and eosinophil proliferation, as well as isotype switching for IgE production [27]. Kyrstsonis et al. found that while IL-4 levels were low (median $4 \mathrm{pg} / \mathrm{ml}$ ) at diagnosis in $75 \%$ of $\mathrm{MM}$ patients and then rose in remission (median $25 \mathrm{pg} / \mathrm{ml}$ ), IL-4 values remained stable during the course of the disease in chemotherapy-resistant patients [28]. In addition, Herrmann et al. observed reduced plasma cell growth in MM patients treated with IL-4, probably by inhibition of endogenous IL-6 synthesis [29].

In our study, a high level of IL-1Ra correlated with shorter OS (Fig. 2). IL-1Ra is an anti-inflammatory acute-phase protein that competitively inhibits IL-1 activity and specifically inhibits paracrine IL-6 production [30,31]. Previous research has found higher levels of IL-1RA to be associated with bone involvement [12], and MGUS/SM/MM patients have demonstrated significant increases in serum IL-1Ra levels compared to healthy controls [32]. In addition, MM patients have been found to demonstrate elevated IL-1Ra levels in the bone marrow (BM) environment [33] and that IL-1Ra is produced by MM cells. Low post-transplantation IL-1Ra levels have also been found to correlate with engraftment syndrome in patients with plasma cell dyscrasias such as POEMS (polyradiculoneuropathy, organomegaly, endocrinopathy, M-spike, skin changes) [34]. IL-1Ra is a specific blocker of IL-1, which is a crucial factor in the induction of IL-17-producing T-cells in vivo [35].

A phase II trial of patients with smoldering or indolent MM showed improvement in PFS and OS duration after targeted treatment with IL-1Ra (Anakinra), with or without dexamethasone [36]. It was found that IL-1Ra bound to the myeloma proliferative cells and decreased the level of C-reactive protein (CRP), a surrogate for IL-6 production. Seven patients treated with IL-RA alone demonstrated a decrease in the plasma cell labeling index (PCLI) and three patients achieved a minor response (MR) to IL-1Ra alone. 
When dexamethasone was added, an additional nine patients achieved a PR/MR. This study suggested that IL-1Ra, as a specific inhibitor of IL-1, induced paracrine IL-6 production and was effective in destroying the proliferative myeloma component.

IL-7 and platelet derived growth factor-BB (PDGF-BB) were also found to be significant predictors of OS in univariate analyses. IL-7 is a cytokine secreted by bone marrow stromal cells. It was previously observed that IL-7 prevents osteoblast formation by decreasing the activity of Runx2/Cbfa1 which is a transcription factor required for osteoblast differentiation [29]. Nierste et al. confirmed the presence of elevated levels of Dickkopf-1 (Dkk-1) and IL-7 in MM patients, and that these were responsible for the osteoblast differentiation from immortalized bone marrow mesenchymal stem cells (MSCs) [37]. In addition, they found that inhibition of Dickkopf-1 (Dkk-1) and IL-7 from MM plasma restored proper osteoblast differentiation in the MSC line. The IL-7 levels did not return to baseline levels in MM patients who are in remission [10].

In the present study, higher platelet-derived growth factor (PDGF)-BB serum level was associated with longer OS in bortezomib-treated patients in univariate analysis. PDGF is an angiogenic factor that can be formed by two A subunits (PDGF-AA), two B subunits (PDGF-BB), or one A and one B (PDGF-AB). PDGF influences c-myc gene expression through the c-myc promoter in a Src-dependent manner [38]. In an in vitro study, PDGF-BB was found to up-regulate Myc expression and reduce the melphalan sensitivity of tumor cell clones. Moreover, down-regulation of c-Myc protein induced the expression of PDGF-beta receptor molecules and decreased PDGF-BB release. Similarly, an in vivo study found melphalan-resistant MM patients to present overexpressed c-Myc protein and higher serum PDGF-BB receptor levels compared to minor responding patients.

The relationship between pretreatment cytokine serum levels and OS in newly-diagnosed MM patients has also been investigated in previous studies. Cheng et al. [39] explored macrophage inflammatory protein 1 alpha (MIP-1 $\alpha$ ), migration inhibitory factor (MIF), tumor necrosis factor- $\alpha$ (TNF- $\alpha)$, vascular endothelial growth factor- $\alpha$ (VEGF- $\alpha$ ), monocyte chemoattractant protein-1 (MCP-1) and soluble interleukins IL-17A, IL-6, IL-21 and IL-10 before treatment. The authors were able to develop a prognostic nomogram using three variables, viz. lactate dehydrogenase (LDH), MIP- $1 \alpha$ and creatinine levels, which accurately predicted the 1-year, 2-year and 3-year OS of MM patients.

Elsewhere, a retrospective analysis by Gu et al. [17] examined the serum levels of seven cytokines in patients with newly-diagnosed MM (IL-2, IL-4, IL-6, IL-10, and IL-17A, TNF- $\alpha$, and IFN- $\gamma$ ). Univariate and multivariate analyses found that that serum IL-6 level $>3 \mathrm{pg} / \mathrm{mL}$, serum IL-17A level $>4 \mathrm{pg} / \mathrm{mL}$ and treatment regimens were independent prognostic factors for PFS and OS. However, IL-4 serum level had no prognostic value, and IL-13 and IL-1RA were not included in the panel of evaluated cytokines in this analysis [17]. In another study, high serum IL-10 was found to predict poor prognosis [40]: the low-IL-10 group ( $\leqslant 169.96 \mathrm{pg} \mathrm{ml}-1)$ was found to have an OR rate of $79.2 \%$ and the high IL-10 group $(>169.96 \mathrm{pg} \mathrm{ml}-1) 53.3 \%(\mathrm{P}<0.001)$. In addition, the patients in low-IL-10 group had significantly better PFS (3-year PFS rate: $69.3 \%$ vs $13.3 \%, \mathrm{P}<0.001$ ) and OS (3-year OS rate: $93.6 \%$ vs $51.9 \%, \mathrm{P}<0.001$ ) than the high-IL-10 group.

Wang et al. found that higher serum level of soluble interleukin-2 receptor (sIL-2R) predicts treatment resistance and poor outcome [41]. The level of sIL-2R in serum was measured using enzyme-linked immunosorbent assay (ELISA) in 81 patients with newly-diagnosed MM; however, in this study, only 26 of 81 (32.1\%) patients included for the analysis were treated with bortezomib-based regimens, and 55 patients $(67.9 \%)$ received older regimens, without targeted drugs. Thirty-six patients (44.4\%) were classified into the higher sIL-2R level group (> $6.049 \mathrm{ng} / \mathrm{ml})$, and 45 patients $(55.6 \%)$ into the lower group. The lower sIL-2R group demonstrated a higher overall response rate (ORR) $(60.0 \%)$, compared to the higher group $(41.7 \%)(p=0.156)$. In addition, the multivariate survival analysis found Eastern Cooperative Oncology Group performance status score, 
treatment response, and sIL-2R level to be significant independent prognostic factors for PFS, but none of these factors was useful for predicting OS. Importantly, a high level of sIL-2R correlated significantly with inferior PFS in patients treated with bortezomib-based regimens $(p=0.004)$. However, sIL-2R was not included in the panel of cytokines investigated in the present study.

The present study also analyzed the influence of clinical variables on PFS and OS in Cox regression models. Of the tested variables, only the use of autologous stem cell transplantation (ASCT) during the treatment schedule was found to influence both PFS and OS. These observations are in agreement with previous reports [42,43]. High-dose therapy with melphalan followed by ASCT prolongs PFS, even in the era of novel agents [44]. A meta-analysis incorporating large phase 3 trials from January 2000 to April 2017 found HDT/ASCT to be associated with superior PFS than standard-dose therapy (SDT). However, the effect of HDT/ASCT on OS remains ambiguous and was not observed in this meta-analysis [44]. In addition, bone disease at diagnosis also significantly impacted PFS, and bone lesions have been found to have a negative prognostic influence in several previous reports [45,46]. A recent meta-analysis of PET parameters found the presence of extramedullary disease, more than three focal lesions, and high FDG uptake to be associated with shorter OS and PFS [47].

\section{Conclusions}

Only IL-13 pretreatment serum level was found to significantly impact PFS in newly-diagnosed MM patients treated with bortezomib-based regimens. In addition, serum levels of five cytokines, IL-1Ra, IL-4, IL-7, IL-13 and PDGF-BB influenced OS in univariate analyses. However, only IL-1Ra and IL-4 were found to have independent prognostic value in multivariate analyses. Three clusters of MM patients were identified, with different cytokine profiles and different OS. Our findings indicate that cytokine signature may have a potential influence on the outcome of MM patients treated with bortezomib. However, the clinical and biological importance of these findings require further investigation.

Supplementary Materials: The following are available online at https://www.mdpi.com/..., Table S1. Cytokine profile of three identified clusters of multiple myeloma patients.; Table S2. Comparison of clinical variables between identified three clusters of multiple myeloma patients.

Author Contributions: P.R., D.M., I.D., J.S. and T.R. designed the study and developed the methodology. P.R., E.W., I.D., D.J. and M.M. performed the experiments. D.M., E.P., A.E., W.F. and T.R. analyzed the data and performed statistical analyses. D.M. and T.R. drafted the manuscript. All authors read and approved the final manuscript.

Funding: This work was supported by a grant from the Narodowe Centrum Nauki (NCN) (2016/23/B/NZ5/02529).

Institutional Review Board Statement: This study was approved by the Ethical Committee of the Medical University of Lodz (No RNN/103/16/KE).

Informed Consent Statement: Informed consent was obtained from the patients included in the study.

Data Availability Statement: The data presented in this study are available from the corresponding author for request.

Acknowledgments: We thank Edward Lowczowski from the Medical University of Lodz for editorial assistance.

Conflicts of Interest: The authors declare no conflict of interest. The funders had no role in the design of the study; in the collection, analyses, or interpretation of data; in the writing of the manuscript, or in the decision to publish the results.

\section{Abbreviations:}

ASCT - autologous stem cell transplantation

$\mathrm{BM}$ - bone marrow 
BM-MSC - bone marrow-derived mesenchymal stem cells

CRAB - calcium elevated, renal failure, anemia, and bone lesions

CXCL1 - C-X-C motif ligand 1IL- interleukin

GM-CSF - granulocyte, monocyte colony stimulating factor

IFN - interferon

IL-1-Ra - IL-1 receptor antagonist

IMiDs - immunomodulatory drugs

IMWG - International Myeloma Working Group

LCD - light chain disease

MIP - macrophage inflammatory protein

MM - Multiple myeloma

MSC - mesenchymal stem cells

OS - overall survival

PC - plasma cells

PCM - plasma cell myeloma

PDGF - platelet derived growth factor

PFS - progression-free survival

PI - proteasome inhibitor

POEMS - polyradiculoneuropathy, organomegaly, endocrinopathy, M-spike, skin changes

RANTES - Regulated upon Activation, Normal T Cell Expressed and Presumably Secreted

TGF- $\beta$ - transforming growth factor $\beta$

TNF- tumor necrosis factor

VCD - bortezomib, cyclophosphamide, dexamethasone

VEGF - vascular endothelial growth factor

VMP - bortezomib, melphalan and prednisone;

VTD -bortezomib, thalidomide, dexamethasone

\section{References}

1. Palumbo, A.; Anderson, K. Multiple Myeloma. N. Engl. J. Med. 2011, 364 (11), 1046-1060. https://doi.org/10.1056/NEJMra1011442.

2. van de Donk, N. W. C. J.; Pawlyn, C.; Yong, K. L. Multiple Myeloma. Lancet 2021, 397 (10272), $410-427$. https://doi.org/10.1016/S0140-6736(21)00135-5.

3. Sung, H.; Ferlay, J.; Siegel, R. L.; Laversanne, M.; Soerjomataram, I.; Jemal, A.; Bray, F. Global Cancer Statistics 2020: GLOBOCAN Estimates of Incidence and Mortality Worldwide for 36 Cancers in 185 Countries. CA. Cancer J. Clin. 2021, 71 (3), 209-249. https://doi.org/https://doi.org/10.3322/caac.21660.

4. Alexander, D. D.; Mink, P. J.; Adami, H.-O.; Cole, P.; Mandel, J. S.; Oken, M. M.; Trichopoulos, D. Multiple Myeloma: A Review of the Epidemiologic Literature. Int. J. Cancer 2007, 120 (S12), 40-61. https://doi.org/https://doi.org/10.1002/ijc.22718.

5. Hemminki, K.; Försti, A.; Houlston, R.; Sud, A. Epidemiology, Genetics and Treatment of Multiple Myeloma and Precursor Diseases. Int. J. Cancer 2021, n/a (n/a). https://doi.org/https://doi.org/10.1002/ijc.33762.

6. Robak, P.; Robak, T. Novel Drugs for Multiple Myeloma. In Topics in Anti-Cancer Research; Atta-ur-Rahman, Zaman, K., Eds.; Topics in Anti-Cancer Research; BENTHAM SCIENCE PUBLISHERS, $2019 ;$ pp 8, 1-43. https://doi.org/10.2174/97898114043821190801.

7. Robak, P.; Robak, T. Bortezomib for the Treatment of Hematologic Malignancies: 15 Years Later. Drugs R. D. 2019, 19 (2), 
73-92. https://doi.org/10.1007/s40268-019-0269-9.

8. Scott, K.; Hayden, P. J.; Will, A.; Wheatley, K.; Coyne, I. Bortezomib for the Treatment of Multiple Myeloma. Cochrane database Syst. Rev. 2016, 4, CD010816. https://doi.org/10.1002/14651858.CD010816.pub2.

9. Robak, P.; Drozdz, I.; Szemraj, J.; Robak, T. Drug Resistance in Multiple Myeloma. Cancer Treat. Rev. 2018, 70, 199-208. https://doi.org/10.1016/j.ctrv.2018.09.001.

10. Zheng, M. M.; Zhang, Z.; Bemis, K.; Belch, A. R.; Pilarski, L. M.; Shively, J. E.; Kirshner, J. The Systemic Cytokine Environment Is Permanently Altered in Multiple Myeloma. PLoS One 2013, 8 (3), e58504-e58504. https://doi.org/10.1371/journal.pone.0058504.

11. Robak, P.; Wẹgłowska, E.; Dróżdż, I.; Mikulski, D.; Jarych, D.; Ferlińska, M.; Wawrzyniak, E.; Misiewicz, M.; Smolewski, P.; Fendler, W.; Szemraj, J.; Robak, T. Cytokine and Chemokine Profile in Patients with Multiple Myeloma Treated with Bortezomib. Mediators Inflamm. 2020, 2020. https://doi.org/10.1155/2020/1835836.

12. Jasrotia, S.; Gupta, R.; Sharma, A.; Halder, A.; Kumar, L. Cytokine Profile in Multiple Myeloma. Cytokine 2020, $136,155271$. https://doi.org/https://doi.org/10.1016/j.cyto.2020.155271.

13. Bębnowska, D.; Hrynkiewicz, R.; Grywalska, E.; Pasiarski, M.; Sosnowska-Pasiarska, B.; Smarz-Widelska, I.; Góźdź, S.; Roliński, J.; Niedźwiedzka-Rystwej, P. Immunological Prognostic Factors in Multiple Myeloma. Int. J. Mol. Sci. 2021, 22 (7), 3587. https://doi.org/10.3390/ijms22073587.

14. Kyle, R. A.; Rajkumar, S. V. Criteria for Diagnosis, Staging, Risk Stratification and Response Assessment of Multiple Myeloma. Leukemia 2009, 23 (1), 3-9. https://doi.org/10.1038/leu.2008.291.

15. Rajkumar, S. V. Updated Diagnostic Criteria and Staging System for Multiple Myeloma. Am. Soc. Clin. Oncol. Educ. B. 2016, No. 36, e418-e423. https://doi.org/10.1200/EDBK_159009.

16. Budczies, J.; Klauschen, F.; Sinn, B. V; Győrffy, B.; Schmitt, W. D.; Darb-Esfahani, S.; Denkert, C. Cutoff Finder: A Comprehensive and Straightforward Web Application Enabling Rapid Biomarker Cutoff Optimization. PLoS One 2012, 7 (12), e51862.

17. Gu, J.; Huang, X.; Zhang, Y.; Bao, C.; Zhou, Z.; Jin, J. Cytokine Profiles in Patients with Newly Diagnosed Multiple Myeloma: Survival Is Associated with IL-6 and IL-17A Levels. Cytokine 2021, 138, 155358. https://doi.org/https://doi.org/10.1016/j.cyto.2020.155358.

18. Stocchi, V.; Wang, T.; Randelli, E.; Mazzini, M.; Gerdol, M.; Pallavicini, A.; Secombes, C. J.; Scapigliati, G.; Buonocore, F. Evolution of Th2 Responses: Characterization of IL-4/13 in Sea Bass (Dicentrarchus Labrax L.) and Studies of Expression and Biological Activity. Sci. Rep. 2017, 7 (1), 2240. https://doi.org/10.1038/s41598-017-02472-y.

19. L, R.; SL, C. IL-13 Regulates Vascular Cell Adhesion Molecule-1 Expression in Human Osteoblasts. J. Cell. Biochem. 2003, 89 (2), 213-219. https://doi.org/10.1002/JCB.10531.

20. Di Lullo, G.; Marcatti, M.; Heltai, S.; Brunetto, E.; Tresoldi, C.; Bondanza, A.; Bonini, C.; Ponzoni, M.; Tonon, G.; Ciceri, F.; Bordignon, C.; Protti, M. P. Th22 Cells Increase in Poor Prognosis Multiple Myeloma and Promote Tumor Cell Growth and Survival. Oncoimmunology 2015, 4 (5), e1005460-e1005460. https://doi.org/10.1080/2162402X.2015.1005460.

21. Bochner, B. S.; Klunk, D. A.; Sterbinsky, S. A.; Coffman, R. L.; Schleimer, R. P. IL-13 Selectively Induces Vascular Cell Adhesion Molecule-1 Expression in Human Endothelial Cells. J. Immunol. 1995, 154 (2), 799 LP - 803.

22. Doucet, C.; Brouty-Boyé, D.; Pottin-Clemenceau, C.; Jasmin, C.; Canonica, G. W.; Azzarone, B. IL-4 and IL-13 Specifically Increase Adhesion Molecule and Inflammatory Cytokine Expression in Human Lung Fibroblasts. Int. Immunol. 1998, 10 (10), 1421-1433. https://doi.org/10.1093/intimm/10.10.1421.

23. Xue, J.; Schmidt, S. V; Sander, J.; Draffehn, A.; Krebs, W.; Quester, I.; De Nardo, D.; Gohel, T. D.; Emde, M.; Schmidleithner, L.; Ganesan, H.; Nino-Castro, A.; Mallmann, M. R.; Labzin, L.; Theis, H.; Kraut, M.; Beyer, M.; Latz, E.; Freeman, T. C.; Ulas, T.; Schultze, J. L. Transcriptome-Based Network Analysis Reveals a Spectrum Model of Human Macrophage Activation. Immunity 2014, 40 (2), 274-288. https://doi.org/10.1016/j.immuni.2014.01.006. 
24. Murray, P. J.; Allen, J. E.; Biswas, S. K.; Fisher, E. A.; Gilroy, D. W.; Goerdt, S.; Gordon, S.; Hamilton, J. A.; Ivashkiv, L. B.; Lawrence, T.; Locati, M.; Mantovani, A.; Martinez, F. O.; Mege, J.-L.; Mosser, D. M.; Natoli, G.; Saeij, J. P.; Schultze, J. L.; Shirey, K. A.; Sica, A.; Suttles, J.; Udalova, I.; van Ginderachter, J. A.; Vogel, S. N.; Wynn, T. A. Macrophage Activation and Polarization: Nomenclature and Experimental Guidelines. Immunity 2014, $41 \quad(1)$, $14-20$. https://doi.org/10.1016/j.immuni.2014.06.008.

25. Alaeddine, M.; Prat, M.; Poinsot, V.; Gouazé-Andersson, V.; Authier, H.; Meunier, E.; Lefèvre, L.; Alric, C.; Dardenne, C.; Bernad, J.; Alric, L.; Segui, B.; Balard, P.; Couderc, F.; Couderc, B.; Pipy, B.; Coste, A. IL13-Mediated Dectin-1 and Mannose Receptor Overexpression Promotes Macrophage Antitumor Activities through Recognition of Sialylated Tumor Cells. Cancer Immunol. Res. 2019, 7 (2), 321 LP - 334. https://doi.org/10.1158/2326-6066.CIR-18-0213.

26. Özyörük, D.; Yavuz, G.; Dinçaslan, H.; Cabı-Unal, E.; Taçyıldız, N.; Karataş, D.; Doğu, F.; İkincioğulları, A. Serum IL-13 Levels at Diagnosis and Remission in Children with Malignant Lymphoma. Turk. J. Pediatr. 2016, 58 (3), $246-253$. https://doi.org/10.24953/turkjped.2016.03.003.

27. Terpos, E.; Dimopoulos, M.-A. Myeloma Bone Disease: Pathophysiology and Management. Ann. Oncol. 2005, 16 (8), 1223-1231. https://doi.org/10.1093/annonc/mdi235.

28. Kyrstsonis, M.-C.; Dedou ssis, G.; Bax evanis, C.; Stamate lou, M.; Maniatis, A. Serum Interleukin-6 (IL-6) and Interleukin-4 (IL-4) in Patients with Multiple Myeloma (MM). Br. J. Haematol. $1996, \quad 92 \quad$ (2), $420-422$. https://doi.org/https://doi.org/10.1046/j.1365-2141.1996.d01-1491.x.

29. Herrmann, F.; Andreeff, M.; Gruss, H. J.; Brach, M. A.; Lubbert, M.; Mertelsmann, R. Interleukin-4 Inhibits Growth of Multiple Myelomas by Suppressing Interleukin-6 Expression. Blood 1991, $78 \quad(8), \quad 2070-2074$. https://doi.org/10.1182/blood.V78.8.2070.2070.

30. Garat, C.; Arend, W. P. Intracellular IL-1Ra Type 1 Inhibits IL-1-Induced IL-6 and IL-8 Production in Caco-2 Intestinal Epithelial Cells through Inhibition of P38 Mitogen-Activated Protein Kinase and NF-KB Pathways. Cytokine 2003, 23 (1), 31-40. https://doi.org/https://doi.org/10.1016/S1043-4666(03)00182-0.

31. Gabay, C.; Lamacchia, C.; Palmer, G. IL-1 Pathways in Inflammation and Human Diseases. Nat. Rev. Rheumatol. 2010, 6 (4), 232-241. https://doi.org/10.1038/nrrheum.2010.4.

32. Bosseboeuf, A.; Allain-Maillet, S.; Mennesson, N.; Tallet, A.; Rossi, C.; Garderet, L.; Caillot, D.; Moreau, P.; Piver, E.; Girodon, F.; Perreault, H.; Brouard, S.; Nicot, A.; Bigot-Corbel, E.; Hermouet, S.; Harb, J. Pro-Inflammatory State in Monoclonal Gammopathy of Undetermined Significance and in Multiple Myeloma Is Characterized by Low Sialylation of Pathogen-Specific and Other Monoclonal Immunoglobulins. Frontiers in immunology. CRCINA, INSERM, Institut de Recherche en Santé 2 (IRS-2), Université de Nantes, Nantes, France. 2017, p 1347. https://doi.org/10.3389/fimmu.2017.01347.

33. Cao, Y.; Luetkens, T.; Kobold, S.; Hildebrandt, Y.; Gordic, M.; Lajmi, N.; Meyer, S.; Bartels, K.; Zander, A. R.; Bokemeyer, C.; Kröger, N.; Atanackovic, D. The Cytokine/Chemokine Pattern in the Bone Marrow Environment of Multiple Myeloma Patients. Exp. Hematol. 2010, 38 (10), 860-867. https://doi.org/10.1016/j.exphem.2010.06.012.

34. Keyzner, A.; D’Souza, A.; Lacy, M.; Gertz, M.; Hayman, S.; Buadi, F.; Kumar, S.; Dingli, D.; Engebretson, A.; Tong, C.; Dispenzieri, A. Low Levels of Interleukin-1 Receptor Antagonist (IL-1RA) Predict Engraftment Syndrome after Autologous Stem Cell Transplantation in POEMS Syndrome and Other Plasma Cell Neoplasms. Biol. Blood Marrow Transplant. 2013, 19 (9), 1395-1398. https://doi.org/10.1016/j.bbmt.2013.06.012.

35. Donovan, K. A.; Moon-Tasson, L. L.; Lust, J. A. Interplay Between IL-1, IL-6 and IL-17 in IL-1 Receptor Antagonist (IL-1Ra) Treated Multiple Myeloma Patients. Blood 2012, 120 (21), 1874. https://doi.org/10.1182/blood.V120.21.1874.1874.

36. Lust, J. A.; Lacy, M. Q.; Zeldenrust, S. R.; Witzig, T. E.; Moon-Tasson, L. L.; Dinarello, C. A.; Donovan, K. A. Reduction in C-Reactive Protein Indicates Successful Targeting of the IL-1/IL-6 Axis Resulting in Improved Survival in Early Stage Multiple Myeloma. Am. J. Hematol. 2016, 91 (6), 571-574. https://doi.org/10.1002/ajh.24352.

37. Nierste, B. A.; Glackin, C. A.; Kirshner, J. Dkk-1 and IL-7 in Plasma of Patients with Multiple Myeloma Prevent 
Differentiation of Mesenchymal Stem Cells into Osteoblasts. Am. J. Blood Res. 2014, 4 (2), $73-85$.

38. Greco, C.; D’Agnano, I.; Vitelli, G.; Vona, R.; Marino, M.; Mottolese, M.; Zuppi, C.; Capoluongo, E.; Ameglio, F. C-Myc Deregulation Is Involved in Melphalan Resistance of Multiple Myeloma: Role of PDGF-BB. Int. J. Immunopathol. Pharmacol. 2006, 19 (1), 205873920601900100. https://doi.org/10.1177/205873920601900107.

39. Cheng, Q.; Zhao, F.; Zhang, B.; Zhang, Y.; Cai, L.; Qiao, B.; Hu, Y.; Sun, C. Prognostic Nomogram Incorporating Cytokines for Overall Survival in Patients with Newly Diagnosed Multiple Myeloma. Int. Immunopharmacol. 2021, $99,108016$. https://doi.org/10.1016/j.intimp.2021.108016.

40. Wang, H.; Wang, L.; Chi, P.-D.; Wang, W.; Chen, X.-Q.; Geng, Q.-R.; Xia, Z.-J.; Lu, Y. High Level of Interleukin-10 in Serum Predicts Poor Prognosis in Multiple Myeloma. Br. J. Cancer 2016, 114 (4), 463-468. https://doi.org/10.1038/bjc.2016.11.

41. Wang, L.; Wang, J.-H.; Liu, W.-J.; Wang, W.; Wang, H.; Chen, X.-Q.; Geng, Q.-R.; Lu, Y.; Xia, Z.-J. High Level of Soluble Interleukin-2 Receptor in Serum Predicts Treatment Resistance and Poor Progression-Free Survival in Multiple Myeloma. Ann. Hematol. 2017, 96 (12), 2079-2088. https://doi.org/10.1007/s00277-017-3125-4.

42. Aggarwal, M.; Agrawal, N.; Yadav, N.; Verma, P.; Ahmed, R.; Mehta, P.; Kapoor, J.; Bhurani, D. Autologous Stem Cell Transplantation in First Remission Is Associated with Better Progression-Free Survival in Multiple Myeloma. Ann. Hematol. 2018, 97 (10), 1869-1877. https://doi.org/10.1007/s00277-018-3370-1.

43. Attal, M.; Harousseau, J. L.; Stoppa, A. M.; Sotto, J. J.; Fuzibet, J. G.; Rossi, J. F.; Casassus, P.; Maisonneuve, H.; Facon, T.; Ifrah, N.; Payen, C.; Bataille, R. A Prospective, Randomized Trial of Autologous Bone Marrow Transplantation and Chemotherapy in Multiple Myeloma. Intergroupe Français Du Myélome. N. Engl. J. Med. 1996, 335 (2), 91-97. https://doi.org/10.1056/NEJM199607113350204.

44. Dhakal, B.; Szabo, A.; Chhabra, S.; Hamadani, M.; D’Souza, A.; Usmani, S. Z.; Sieracki, R.; Gyawali, B.; Jackson, J. L.; Asimakopoulos, F.; Hari, P. N. Autologous Transplantation for Newly Diagnosed Multiple Myeloma in the Era of Novel Agent Induction: A Systematic Review and Meta-Analysis. JAMA Oncol. 2018, 4 (3), 343-350. https://doi.org/10.1001/jamaoncol.2017.4600.

45. Jung, S.-H.; Kwon, S. Y.; Min, J.-J.; Bom, H.-S.; Ahn, S.-Y.; Jung, S.-Y.; Lee, S.-S.; Park, M.-R.; Yang, D.-H.; Ahn, J.-S.; Kim, H.-J.; Lee, J.-J. (18)F-FDG PET/CT Is Useful for Determining Survival Outcomes of Patients with Multiple Myeloma Classified as Stage II and III with the Revised International Staging System. Eur. J. Nucl. Med. Mol. Imaging 2019, 46 (1), 107-115. https://doi.org/10.1007/s00259-018-4114-0.

46. Abe, Y.; Narita, K.; Kobayashi, H.; Kitadate, A.; Takeuchi, M.; O'uchi, T.; Matsue, K. Medullary Abnormalities in Appendicular Skeletons Detected With (18)F-FDG PET/CT Predict an Unfavorable Prognosis in Newly Diagnosed Multiple Myeloma Patients With High-Risk Factors. AJR. Am. J. Roentgenol. 2019, 213 (4), 918-924. https://doi.org/10.2214/AJR.19.21283.

47. Han, S.; Woo, S.; Kim, Y.-I.; Yoon, D. H.; Ryu, J.-S. Prognostic Value of \&lt;Sup\&gt;18\&lt;/Sup\&gt;F-Fluorodeoxyglucose Positron Emission Tomography/Computed Tomography in Newly Diagnosed Multiple Myeloma: A Systematic Review and Meta-Analysis. Eur. Radiol. 2021, 31 (1), 152-162. https://doi.org/10.1007/s00330-020-07177-x.

\section{Supplementary tables:}

Table S1. Cytokine profile of three identified clusters of multiple myeloma patients. Data are presented as mean values and SD, and as p-values from the global ANOVA test and post hoc comparisons (Tukey's test), if the p-value of the global test is significant.

Table S2. Comparison of clinical variables between identified three clusters of multiple myeloma patients. 